\title{
Clinician-Patient Small Talk: Comparing Fourth-Year Dental Students and Practicing Dentists in a Standardized Patient Encounter
}

\begin{abstract}
Gerardo Maupome, BDS, MSc, PhD; Christopher Holcomb, BSc; Stuart Schrader, PhD
Abstract: The aim of this study was to establish whether frequency of non-diagnostic, non-management exchanges between clinicians and patient (called "socioemotional communication," SC) during a consultation differed between fourth-year dental students and dentists, controlling for clinically driven exchanges of information. Fifteen dentists and 17 fourth-year dental students were recorded in 2006 while undergoing a consultation with a live standardized patient and were subsequently interviewed by investigators in a separate room with the recording present for analysis. Their shared interpretations of cognitive strategies were recorded and compared for differences in the presence of SC. The results showed that most of the students and dentists engaged in SC throughout the consultation with a few exceptions. There were no significant differences between student and dentist cohorts for overall SC presence ( $\mathrm{p}=0.62$ ), time to first instance of SC from overall start of the consultation ( $\mathrm{p}=0.73$ ), and time to first instance of SC after first intraoral examination had taken place $(\mathrm{p}=0.76)$. Nonsignificant differences were also recorded for overall frequency at which $\mathrm{SC}$ occurred from overall start of the consultation $(\mathrm{p}=0.89)$ and after the first intraoral examination had taken place $(\mathrm{p}=0.12)$. The patterns showed SC interaction occurring throughout the consultation, not concentrated at the beginning or end. SC did not appear to differ between practitioners and students in terms of prevalence, frequency, or timing. Future research should examine the detailed association between SC and diagnostic thinking processes to further delineate the relationship and characterize possible pedagogical applications.
\end{abstract}

Dr. Maupome is Professor, Department of Cariology, Operative Dentistry, and Dental Public Health, Indiana University School of Dentistry; Mr. Holcomb is a dental student, Indiana University School of Dentistry; and Dr. Schrader is Associate Professor, Department of Biomedical and Applied Sciences, Indiana University School of Dentistry. Direct correspondence to Dr. Gerardo Maupome, Department of Cariology, Operative Dentistry, and Dental Public Health, Indiana University School of Dentistry, 1121 West Michigan Street, Indianapolis, IN 46202; gmaupome@iu.edu.

Keywords: dental education, patients, patient-centered care, doctor-patient relations, clinical decision making, socioemotional communication, psychosocial aspects

Submitted for publication 2/3/16; accepted 4/19/16

$\mathrm{D}$ ental education is primarily about creating competent dental practitioners. This process is realized by educating students in the technical skills and scientific knowledge necessary to effectively manage clinical cases. It also requires purposeful development of communication skills to facilitate an effective exchange between clinician and patient about diagnoses, treatment plans, health education, and oral health management. ${ }^{1}$ Communication skills and process training regarding communicative strategies, relationship-building, cultural appropriateness, and empathy have markedly increased over the years in dental education; however, they still remain secondary to technical skills training. ${ }^{2-6}$ Overall, dental education aims to move novice dental students toward expert overall proficiency in providing oral health care services.

Although novice and expert dental providers share many attributes, there are key differences in the frequency of cognitive strategies used to process clinical information and in the communicative content associated with these cognitive strategies. ${ }^{7}$ Understanding the subtle differences in cognitive strategies and use of communicative content would benefit dental education by informing educators how to more effectively graduate competent students who efficiently gather and process clinical information. Prior research about clinical practice has proposed a critical pathway that involves the clinician interactively interviewing the patient, cognitively processing the presenting problem(s), presenting a diagnosis, giving advice, delivering treatment plans, and reviewing patient progress. ${ }^{8}$ Several studies have found dentists become increasingly proficient in their clinical reasoning and decision making processes as a result of repeatedly executing these clinical communication actions. ${ }^{7,-12}$ This clinical focus fits well with a perspective suggesting there are three general communicative purposes during a clinical encounter: creating a positive interpersonal relationship, exchanging clinical information, and making treatment-related decisions. ${ }^{8}$ Socioemotional 
communication may have some role in fulfilling all of these purposes, but mostly it assists both participants in building their interpersonal relations. Interpersonal communication is a "complex, situated social process in which people who have established a communicative relationship exchange [verbal and nonverbal] messages in an effort to generate shared meanings and accomplish social goals." 13

There has been far less research focusing on the communicative spaces in provider and patient exchanges that are not centered on gathering, processing, or sharing of clinical information. All doctor-patient communication routinely plays a role in diagnosing and providing treatment. ${ }^{14}$ Even exchanges that seemingly lack a direct clinical focus are essential in relationship-building and trust-gaining so that both parties can seek more information from the other. ${ }^{15}$ These forms of communicative spaces are called "socioemotional communication" (SC).${ }^{16} \mathrm{SC}$ is similar to but more comprehensive than small talk and refers to care-oriented interactions that may have the intended purposes of making the patient feel comfortable, building relationships, and developing trust. It could include a dentist or patient being sympathetic, empathetic, concerned, reassuring, or friendly; it may also involve greetings and active listening.

SC serves a variety of communicative purposes. One common purpose is humor. Informal pleasantry may more often occur at the beginning of clinical interviews involving casual communication. Ultimately, relationship building focuses on personal talk that relationally helps to connect the two parties aside from their roles as patient and doctor. Additionally, sequences related to clinical small talk like SC include apologizing and being accepting, exchanging appreciations and acknowledgments, and providing greetings. SC includes conversational examples such as "greeting each other by first names," "a patient asking a dentist about a son's schooling," "a patient joking with the dentist about how he/she couldn't catch any fish on vacation," "both people teasing each other about having their kids go off to college," "talk about vacations, exercise routines, getting divorced, playing baseball, liking of certain TV shows, personal family conflicts, or asking about one's new baby."17 Amounts of SC in doctor-patient interactions may vary greatly. One study found that the greater the frequency of SC, the stronger the social relations between doctor and patient, and the more likely the two are to develop a trusting bond that may support clinical decision making. ${ }^{16}$ How is this conversational segment of clinical dialogue likely to operate?
A clinician processes not only the essential, obvious, and explicit diagnostic information to develop a patient's medical, dental, social, family, and medication history; he or she is also using the mutually exchanged SC to help place such diagnostic clinical decision making information within the shared relationship. SC may also be applied to evaluate the likelihood that a patient will successfully follow recommendations; for example, how good the patient's adherence will likely be to comply with a treatment plan. Crespo et al. proposed that such a form of clinical communication provides the social and relational context needed for effectively engaging in various diagnostic processes. ${ }^{9}$ For example, a clinician extrapolates a patient's demeanor about dental anxiety by listening to the patient share uneasiness or even panic when getting a flu vaccine. The clinician may then deduce from such apprehension a general condition of anxiety for this patient because of prior experience with needles. If the same patient added that he or she "freaks out" in elevators or planes, it may act as an additional indicator of a more clinically defined form of anxiety or even an anxiety disorder.

The aim of our study was to examine the frequency of SC during a dental consultation by analyzing data from a prior study comparing practicing dentists and students involved in clinical decision making. ${ }^{7}$ That prior study adopted a developmental perspective to characterize the similarities and differences between fourth-year dental students and experienced dental clinicians' cognitive psychological processes. Both groups' diagnostic thinking processes were examined by quantifying the patterns based on analysis of a consultation with a live standardized patient who presented with the chief complaint of needing a "broken tooth fixed and a cleaning." By holding constant the clinical focus of the patient-clinician exchanges, we isolated SC and characterized its occurrences. The research question asked if there was a difference between these dental students and dentists in the frequency of SC during the live standardized patient dental encounter.

\section{Materials and Methods}

The study was approved by the Indiana University School of Dentistry Institutional Review Board (IRB 0606-57). In a previous study, we used an established model to identify the cognitive strategies used in diagnostic thinking. ${ }^{7}$ The current study makes 
further use of the data from that study, collected in 2006. Gale and Marsden explained the dynamics of clinical decision making by identifying cognitive processes that occur as the clinician moves through the resolution of a clinical problem. ${ }^{18}$ These cognitive processes are called Diagnostic Thinking Processes (DTP). Table 1 outlines the DTPs used in the previous study. ${ }^{7}$ Gale and Marsden described their model as an adjustable set of strategies, which depends upon the interpretation of a diagnostic challenge. ${ }^{18}$ Clini- cians' knowledge and their understanding of how to develop diagnoses vary from person to person. The link among clinical knowledge, strategies, and a specific clinical situation is derived from cognitive pathways that link the clinician's knowledge and a particular situation. ${ }^{19-21}$ No significant differences have been found in the range of DTPs available to experienced or student clinicians. ${ }^{22}$ Gale and Marsden interpreted this finding by suggesting that differences in expertise are mainly differences in the content

\section{Table 1. Inventory of diagnostic thinking processes (DTPs) in Gale and Marsden model of clinical decision making}

DTP 1: Pre-diagnostic interpretation of clinical information.

Any term that indicates the clinician has made some interpretation of the information available when the result of this activity is not sufficiently specific to constitute a diagnosis.

DTP 2: Diagnostic interpretation of clinical information. Same as DTP 1 but with a greater degree of specificity that is sufficient for a diagnosis.

DTP 3: Judgment of need for further general or clarifying inquiry, not stemming from either prediagnostic or diagnostic interpretations. When the clinician inquires further about the patient's symptoms, signs, etc. for clarification.

DTP 4: Expecting, searching for, or planning to search for specific features of disease or treatment of disease. When the clinician shows expectation of information or considers likely certain features of disease, given the information already obtained.

DTP 5: Reinterpretation of clinical information, when no new information has been added. When an array of clinical information already interpreted in some way becomes amenable to a new (altered or additional) interpretation because of a change in the clinician's own thinking and not because new information has been added. The new interpretation may or may not be related to the old one(s).

DTP 6: Reinterpretation of clinical information arising from addition of new information. Same as DTP 5 but stemming from addition of new information.

DTP 7: Inquiry responsive to elicited information. When the course of the visit as directed by the clinician is determined by, or follows from, the flow of information as presented by the patient.

DTP 8: Inquiry determined by the clinician's interpretation.

When the course of the visit is determined by the clinician's requirement to test his/her active interpretation of the clinical information.

DTP 9: Routine inquiry.

When the clinician conducts the visit according to a routine format as defined by the standard clinical history or any of its components.

DTP 10: Failure to make specific inquiry.

When the clinician identifies, in retrospect, his/her own failure to make a specific inquiry concerning patient's problem, symptoms, signs, etc.

DTP 11: Failure to make general inquiry.

When the clinician identifies, in retrospect, his/her own failure to make sufficient routine, general, or screening inquiry.

DTP 12: Active confirmation of an interpretation.

When the clinician feels that the selected interpretation is confirmed as an actual diagnosis.

DTP 13: Active elimination of an interpretation.

When the clinician eliminates an identified interpretation because of contrary evidence or lack of necessary evidence.

DTP 14: Postponement of either confirmation or elimination of an interpretation with or without stated differential likelihoods. When an interpretation is neither confirmed nor eliminated but is left under postponed judgment.

Note: Items are authors' summaries of material from Gale J, Marsden P. Medical diagnosis: from student to clinician. New York: Oxford University Press, 1983; and Gale J, Marsden P. Clinical problem solving: the beginning of the process. Med Educ 1982;16:22-6. 
and structure of memory, but not due to differences in the thought processes. ${ }^{18}$ Our prior results largely supported their interpretation. ${ }^{7}$

In order to better understand the DTPs of students versus practicing dentists, it was necessary to find a "typical" dental patient, who could be an adult of either gender, to act as the standardized patient in the encounters. This person was dentate, with some restorative and periodontal treatment needs, but free of major prosthetic, surgical, or medical issues, and the person was interested in entering dental care as a new patient. Full details are in the previous article. ${ }^{7}$ One individual was selected and trained, and a panoramic film radiograph and a radiographic series were taken. Full periodontal charting was conducted, and study models were fabricated. All information was entered into an electronic dental record system. Additionally, a dental, medical, and psychosocial history was developed, largely reflecting the features of the person acting as the standardized patient. Intraoral images were also collected of each tooth and subsequently serialized in a laptop presentation.

\section{Dental Visit Procedures and Interview Method}

Fourth-year dental students were recruited for the study through open advertising via the Indiana University School of Dentistry listservs, flyers, and word of mouth. IRB-approved language to ensure confidential use of information and complete separation of research from academic grading were emphasized. Dentists were recruited through announcements and email messages addressed to members of the American College of Dentists, inviting them to take part in the study as a research project. The dentists were not dental school faculty members, fullor part-time, but rather engaged full-time in general dental practice. The dental students and practicing dentists were compensated for participation with $\$ 100$ or three hours of continuing education credits, respectively. All 15 dentists and 17 fourth-year dental students who volunteered were accepted as participants in the study.

Prior to the encounter, each clinician was supplied with a description of how the standardized patient consultation could unfold by explaining the charts available and the description of what clinical data he or she was expected to prepare. These clinicians also learned in advance about the overall goals of the dental visit and the agenda for the post- clinical encounter research interviews. Video- and audiorecording equipment was used to record the actual dental visit (called "Tape 1") until it was terminated by the clinician. A researcher observing the encounter in the same dental operatory kept track of the frequency of social interactions between the clinician and patient, noting potential decision making moments.

The clinician and researcher then moved to a separate office after the clinician indicated that he or she was satisfied with the clinical information gathered, and a treatment plan was outlined. Tape 1 of the dental visit was replayed for the researcher and clinician. The researcher interviewed the clinician about his or her clinical decision making based on what both recalled from the consultation, radiographs, medical and dental history, and intraoral images of teeth and mouth. A second video- and audiorecording was then completed (called "Tape 2") as the clinician verbally recalled his or her thoughts. These thoughts were aided by prompts and moments of shared discussion with the researcher. This exchange was intended to gather an account of the clinical strategies pursued and the relevant pieces of information used in the consultation.

Thematic (content) analysis was conducted of the discourse for Tapes 1 and 2 and the tapes themselves. ${ }^{23,24}$ Briefly, this process involves an enumeration of the items of interest in the language and concepts derived from the consultation, using a classification that encompasses the themes of interest. The analysis was intended to link verbal content and nonverbal cues within and between tapes so as to identify a DTP, either by recognizing the verbal/ written content present or by interpreting the clinician's nonverbal cues and interactions. ${ }^{10,25}$ All of those exchanges that could not be ascribed to the 14 DTPs were categorized as SC.

Additionally, a subsequent analysis was conducted of 20 of the audiorecordings, featuring ten fourth-year students (seven male, three female) and ten dentists (five male, five female). Inductive qualitative analytical techniques were used to secondarily analyze a constructed coding scheme for the non-DTP categories so as to explore the language content of the non-clinically focused SC interactions between clinicians and patient. ${ }^{26} \mathrm{We}$ undertook this exercise in only 20 recordings out of a total 32 because we reached data saturation at 20 randomly chosen recordings. 


\section{Statistical Analyses}

We used standard research approaches to quantify thematic findings; results about DTPs and their associated concepts were previously published. ${ }^{7}$ Content-analysis reliability was evaluated by establishing the stability (intracoder variability) of DTP and $\mathrm{SC}$ coding ${ }^{27}$ in four randomly selected interviews blindly recoded. A content-analysis coefficient (Scott's $\pi$ ) was calculated to correct for expected chance agreement. ${ }^{28}$ Stability coefficients for DTP and SC coding ranged from 0.63 to 0.71 . This range is considered appropriate since Scott's $\pi$ is a very conservative assessment.

Data analysis for the research question was based on 1) identifying all exchanges of information between clinician and patient during the consultation and 2) subsequently limiting the focus to a subset of exchanges that could not be accounted for under the previously identified definitions of DTPs in the Gale and Marsden model. All previously identified DTPs were set aside using a rigorous approach of identification and characterization. ${ }^{7}$ The remaining communicative exchanges were $\mathrm{SC}$, as not being primarily driven by a clinician's active search or interpretation of clinically meaningful information. Dentist and student groups were compared for differences in their SC frequency using a chi-square test. Log rank tests were used to assess the occurrences of SC at the beginning of the consultations, and a mixed model ANOVA was conducted for determining the number of overall times SC occurred for dentists and students.

\section{Results}

The 15 dentists (33\% female) and 17 senior dental students ( $47 \%$ female) participated in dental visits lasting mean 32.0 minutes $( \pm 12.9)$ for dentists and $29.9 \pm 7.1$ for students. The length of time between when clinicians first began reviewing patient information and when they requested having the patient brought into the operatory was 5.0 minutes (6.0 \pm 4.9 minutes for dentists, $7.6 \pm 3.7$ for students; $\mathrm{p}=0.20$ ). The face-to-face dental visit time spent with the standardized patient was $26.4 \pm 13.9$ minutes for dentists and $22.2 \pm 7.5$ for students $(p=0.25)$. A mean of $10.8 \pm 6.4$ minutes elapsed for the dentists between the first instance of looking into the mouth of the patient and the last instance. These intervals for first looking into the patient's mouth were not statistically significantly different between the dentists (10.8 16.4$)$ and students (13.2 \pm 5.1$)$. However, substantial variations in time did exist across individual clinicians. As reported in our previous study, the main differences found in DTP utilization showed that dentists conducted diagnostic interpretations of information with sufficient certainty twice as often as did the students, and the students used general or clarifying inquiries in their search for information more often than did the dentists. ${ }^{7}$

We found that most of the clinicians (both students and dentists) engaged in SC throughout the consultation; the exceptions were Students \#15 and \#22 and Dentist \#18. There was substantial variation in the frequency of this form of interpersonal exchange at the individual level. Considering the study used only one standardized patient, all variations were more likely ascribable to the clinician than to the patient. SC exchanges were found throughout the consultation.

Our analysis found negligible differences between the dentist and student groups (Table 2). Differences were not significant for several contrasts: the overall presence of SC (14 dentists used it at least once, one did not; 15 students used it at least once, two did not; $\mathrm{p}=0.62$ ); time to first instance of SC from overall start of the consultation $(\mathrm{p}=0.73)$; and time to first instance of SC after the first intraoral examination had taken place $(p=0.76)$. Non-significant differences were also recorded for overall frequency when SC occurred from overall start of the consultation $(\mathrm{p}=0.89)$ and after the first intraoral examination had taken place $(\mathrm{p}=0.12)$ (Table 3$)$.

Inductive qualitative analysis of 20 recordings identified several features. First, the clinicians and the patient both showed an interest in each other during the opening of the clinical interview, asking questions like "Where are you from in Florida?" Second, they oriented to each other's lives in finding commonality. For example, there were examples of conversations about books read (e.g., the Harry Potter novels) or games played (e.g., games on PlayStation) and connections made about the numbers of family members.

Finally, the use of humor was key throughout the visit. When clinicians and the patient were not engaged in a greeting or seeking a relational orientation or in gaining general informal and social information, they were most often joking. Most of the joking seems to have served a variety of purposes such as to relieve anxiety by calling for practitioner reassurance (e.g., in worrying about her not wishing to necessarily fix her broken tooth, the patient joked, "Did you find my fake filling? It's my own 
Table 2. Time between first instance of socioemotional communication (SC) and clinician's first examination of case information and first contact between clinician and patient, by group

\begin{tabular}{lccccccc} 
Time & $\begin{array}{c}\text { Clinician } \\
\text { Group }\end{array}$ & $\begin{array}{c}\text { Number } \\
\text { in Group }\end{array}$ & $\begin{array}{c}\text { Minimum } \\
\text { Time }\end{array}$ & $\begin{array}{c}\text { 25th } \\
\text { Percentile }\end{array}$ & $\begin{array}{c}\text { Median } \\
\text { Time }\end{array}$ & $\begin{array}{c}\text { 75th } \\
\text { Percentile }\end{array}$ & $\begin{array}{c}\text { Max } \\
\text { Time }\end{array}$ \\
\hline $\begin{array}{l}\text { From clinician's first examination of } \\
\text { case information to first instance of SC }\end{array}$ & Dentists & 15 & 0 & 2 & 8 & 14 & 26 \\
$\begin{array}{l}\text { From first clinician-patient contact to } \\
\text { first instance of SC }\end{array}$ & Dentists & 17 & 2 & 5 & 9 & 11 & 42 \\
& Students & 15 & 0 & 0 & 1 & 4 & 26 \\
\end{tabular}

Note: Time is in minutes. No significant differences existed between dentists and students.

Table 3. Frequency of socioemotional communication (SC) instances and timing of SC, by group

\begin{tabular}{|c|c|c|c|c|c|c|c|c|}
\hline Time & $\begin{array}{l}\text { Clinician } \\
\text { Group }\end{array}$ & $\begin{array}{l}\text { Number of } \\
\text { SC Instances }\end{array}$ & $\begin{array}{l}\text { Mean } \\
(\mathrm{SD})\end{array}$ & $\begin{array}{l}\text { Minimum } \\
\text { Time }\end{array}$ & $\begin{array}{c}\text { 25th } \\
\text { Percentile }\end{array}$ & Median & $\begin{array}{c}\text { 75th } \\
\text { Percentile }\end{array}$ & Max \\
\hline From clinician's first examination & Dentists & 44 & $12.2(9.7)$ & 0 & 5 & 10.5 & 17.5 & 45 \\
\hline of case information to SC & Students & 43 & $12.3(6.0)$ & 2 & 8 & 11 & 16 & 24 \\
\hline $\begin{array}{l}\text { From first clinician-patient contact } \\
\text { to SC }\end{array}$ & $\begin{array}{l}\text { Dentists } \\
\text { Students }\end{array}$ & $\begin{array}{l}44 \\
43\end{array}$ & $\begin{array}{l}8.2(9.2) \\
5.3(5.5)\end{array}$ & $\begin{array}{l}0 \\
0\end{array}$ & 1.5 & $\begin{array}{l}5 \\
3\end{array}$ & $\begin{array}{c}10.5 \\
9\end{array}$ & $\begin{array}{l}37 \\
22\end{array}$ \\
\hline
\end{tabular}

Note: Time is in minutes. There were no significant differences between dentists and students.

artwork," or in seeking reassurance that the dentist would not extract her third molars, she joked, "I always brag because I got to keep my wisdom teeth when everyone had theirs taken out"). Additionally, joking seems to have been initiated by both parties to reduce embarrassment (e.g., a dentist would joke regarding his embarrassment for not being familiar with the operatory by saying, "It's my first day in my new office," or "You're gonna go for a little ride while I figure this [dental chair] out"). Finally, the clinicians responded sometimes with quips to help reduce the patient's uncertainty about why diagnostically something may have happened or to explain that even doing something does not guarantee a specific outcome (e.g., they said, "Your temporary filling came out when you were eating sugar-free candy of course!" and "My husband [also] flosses every day, but of course he has 15 crowns").

\section{Discussion}

This study is one of few research reports examining and quantifying the social communicative interactions between clinicians and patients within a clinical encounter. Our findings were consistent with those of Desjarlais-deKlerk and Wallace that $\mathrm{SC}$ is pervasive throughout the clinical encounter yet highly variable across clinicians. ${ }^{16} \mathrm{SC}$ 's integra- tion throughout the encounter could be an indicator of positivity and a form of balance that is needed in forming genuine rapport between the parties. ${ }^{29}$

In applying a cognitive developmental framework for identifying communicative practices, our qualitative study found no significant variation between the practicing dentists and fourth-year dental students. It may have been expected that differences would be seen between the students and dentists for three possible reasons: enhanced prevalence of communication skills training as part of dental education in the past 15 years and thus more likely to have had an effect on the students; the students' greater comfort with the physical operatory surroundings at the research location (including the possibility of students' being more familiar with computer systems and characteristics of a dental school environment than the typical setting for the dentists); and the students' comfort with standardized patients gained through pedagogical experiences such as objective structured clinical exams (OSCEs). However, those factors did not lead to detectable differences in SC utilization. The magnitude of SC or their patterns of utilization were, in fact, not different for the students and the dentists. This finding may indicate that the role modeling this dental school (and others) uses in clinical education is succeeding in giving students an array of practical tools to engage patients. Whether this learning is part of a hidden curriculum 
or an area amenable to systematic improvement as a pedagogic component of clinical training remains to be addressed in future research.

This study did find that SC was pervasive throughout both student-patient and dentist-patient clinical encounters. This SC prevalence suggests that clinical interviews are not simply explicit clinical information-gathering or -processing communicative interactions. While other research has reported that small talk was not part of the "real" and "important" parts of conversations, ${ }^{30}$ such marginalization of small talk characterized it as inconsequential and possibly informal and outside of normal conversation. Patients may instead use SC for non-formal reasons such as becoming distracted from treatment plans to reduce their anxiety or uncertainty or even to indicate a need of reassurance from their dental providers. ${ }^{31}$ There is clearly a case for SC playing an instrumental role in clinical talk by assisting with diagnosing or treatment planning. Our results suggest that SC, therefore, is not simply at the other end of the continuum from "work" or "clinical" talk ${ }^{31}$ but instead is integrative and used either formally or informally and for purposeful or distractive reasons. It would seem important to continue this line of study to more carefully examine the subtypes and forms of SC and its intended purposes.

This study had limitations. It was a qualitative assessment of clinician-patient interaction, inherently concerned with identifying the features making up the mosaic of communicative processes but not intended to attain an exhaustive quantification of those features. It was not a complete description that can be extrapolated directly to other populations, as it was not undertaken with representative samples. The high fidelity standardized patient situation rendered the object of the consultation highly stable and replicable across the participating clinicians. However, it would be ethically infeasible to create a unique consultation experience in which clinicians would not be aware of the quasi-experimental conditions. As reported in our previous study, the participants indicated in an exit evaluation that they felt their performance in the study was similar to their usual behaviors and approaches in everyday care. ${ }^{7}$ But they also asserted they were always aware of the research conditions, which supports both realism and self-awareness. Finally, the design of the standardized patient explicitly focused on an "average" patient presenting for the first time seeking care, who lacked major complexities or significant barriers in access to care. SC may differ when patients present with urgent treatment needs or insurmountable challenges requiring prioritizing needed treatment services.

\section{Conclusion}

This study found that SC occurred among most of the clinicians (whether practicing dentists or fourth-year dental students) under quasi-experimental conditions in a dental consultation with a standardized patient. The patterns showed that SC interaction took place throughout the consultation and was not necessarily concentrated at the beginning or end of the consultation. More importantly, such interactions did not appear to differ between dentists and students in terms of prevalence, frequency, or timing. Future research should use these findings to develop a survey for formal evaluation of its psychometric properties; such a tool could allow for a larger scale evaluation of SC practices among students or practitioners. Further delineation of the SC components in the context of clinical decision making could allow for the evaluation of functions of SC moments in the consultation that are located in between clinically meaningful strategies aimed at managing care. Future research should examine the detailed association of SC with DTPs to ascertain if and how they relate to each other. Demonstration of those SC-DTP links to students in standardized conditions or OSCE settings could then be evaluated for pedagogical impact during training to enhance the acquisition of clinical decision making skills.

\section{Acknowledgments}

This research was supported in part by a fellowship grant from the Indiana University Center for Research and Learning and by a grant from the American Dental Association Foundation. We also wish to offer considerable gratitude to our colleagues who participated in the design and development of earlier research related to this study.

\section{Disclosure}

The authors declared no conflicts of interest.

\section{REFERENCES}

1. Commission on Dental Accreditation. Accreditation standards for dental education programs. Chicago: American Dental Association, 2013.

2. Yoshida T, Milgrom P, Coldwell S. How do U.S. and Canadian dental schools teach interpersonal communication skills? J Dent Educ 2002;66(11):1281-8. 
3. Freeman R. A psychodynamic understanding of the dentist-patient interaction. Br Dent J 1999;186:503-6.

4. Karydis A, Komnoli-Kodovazeniti M, Hatzigeorgiou D, et al. Expectations and perceptions of Greek patients regarding the quality of dental health care. Int J Qual Health Care 2001;13:409-16.

5. Chant S, Jenkinson T, Randle J, et al. Communication skills: some problems in nursing education and practice. J Clin Nurs 2002;11:12-21.

6. Newsome PRH, Wright GH. A review of patient satisfaction, 2: dental patient satisfaction - an appraisal of recent literature. Br Dent J 1999;186:166-70.

7. Maupome G, Schrader S, Mannan S, et al. Diagnostic thinking and information used in clinical decision making: a qualitative study of expert and student dental clinicians. BMC Oral Health 2010;10:11.

8. Sondell K, Soderfeldt B. Dentist-patient communication: a review of relevant models. Acta Odontol Scand 1997;55:116-26.

9. Crespo KE, Torres JE, Recio ME. Reasoning process characteristics in the diagnostic skills of beginner, competent, and expert dentists. J Dent Educ 2004;68(12):1235-44.

10. Maupome G, Sheiham A. Clinical decision making in restorative dentistry: content analysis of diagnostic thinking processes and concurrent concepts used in an educational environment. Eur J Dent Educ 2000;4:143-52.

11. White BA, Maupome G. Making clinical decisions for dental care: concepts to consider. Spec Care Dent 2003; 23(5):168-72.

12. White BA, Maupome G. Clinical decision making for dental caries management. J Dent Educ 2001;65(10): 1121-5.

13. Burleson BR. The nature of interpersonal communication: a message-centered approach. In: Berger C, Roloff M, Roskos-Ewoldsen D, eds. The handbook of communication science. $2^{\text {nd }}$ ed. Thousand Oaks, CA: Sage, 2010.

14. Arora NK. Interacting with cancer patients: the significance of physicians' communication behavior. Soc Sci Med 2003;57(5):791-806.

15. Becker G, Kempf DE, Xander CJ, et al. Four minutes for a patient, twenty seconds for a relative: an observational study at a university hospital. BMC Health Serv Res 2010;10:94.
16. Desjarlais-deKlerk K, Wallace JE. Instrumental and socioemotional communications in doctor-patient interactions in urban and rural clinics. BMC Health Serv Res 2013;13:261.

17. Maynard DW, Hudak PL. Small talk, high stakes: interactional disattentiveness in the context of prosocial doctorpatient interaction. Lang Society 2008;37(5):661-88.

18. Gale J, Marsden P. Medical diagnosis: from student to clinician. New York: Oxford University Press, 1983.

19. Thyne JM. The psychology of learning and techniques of teaching. London: University of London Press, 1966.

20. Page G, Bordage G, Allen T. Developing key-feature problems and examinations to assess clinical decision making skills. Acad Med 1995;70(3):194-201.

21. Gale J, Marsden P. Clinical problem solving: the beginning of the process. Med Educ 1982;16:22-6.

22. Grant J, Marsden P. The structure of memorized knowledge in students and clinicians: an explanation for diagnostic expertise. Med Educ 1987;21:92-8.

23. Holsti OR. Content analysis. In: Lindzey G, Aronson E, eds. Handbook of social psychology, vol. 2: research methods. Reading, MA: Addison-Wesley, 1968.

24. Weber RP. Basic content analysis. Newbury Park, CA: Sage, 1985.

25. Maupome G, Sheiham A. Explanatory models in the interpretations of clinical features of dental patients within a university dental education setting. Eur J Dent Educ 2002; 6(1):2-8.

26. Strauss A, Corbin J. Basics of qualitative research: grounded theory procedures and techniques. Thousand Oaks, CA: Sage, 1990.

27. Krippendorff K. Content analysis: an introduction to its methodology. Newbury Park, CA: Sage, 1980.

28. Holsti OR. Content analysis for the social sciences and humanities. Reading, MA: Addison-Wesley, 1969.

29. Cappella J. On defining conversational coordination and rapport. Psychol Inquiry 1990;1(4):303-5.

30. Coupland J. Small talk. Harlow: Pearson Education Limited, 2000.

31. Hudak PL, Maynard DW. An interactional approach to conceptualizing small talk in medical interactions. Socio Health Ill 2011;33(4):634-53. 